Article

\title{
Poly $(N$-isopropylacrylamide) Hydrogels for Storage and Delivery of Reagents to Paper-Based Analytical Devices
}

\author{
Haydn T. Mitchell, Spencer A. Schultz, Philip J. Costanzo and Andres W. Martinez * \\ Department of Chemistry and Biochemistry, California Polytechnic State University, 1 Grand Avenue, \\ San Luis Obispo, CA 93407, USA
}

* Author to whom correspondence should be addressed; E-Mail: awmartin@calpoly.edu.

Academic Editor: Carlos D. Garcia

Received: 4 May 2015 / Accepted: 10 July 2015 / Published: 17 July 2015

\begin{abstract}
The thermally responsive hydrogel $N, N^{\prime}$-methylenebisacrylamide-cross-linked poly(N-isopropylacrylamide) (PNIPAM) was developed and evaluated as a reagent storage and delivery system for microfluidic paper-based analytical devices (microPADs). PNIPAM was shown to successfully deliver multiple solutions to microPADs in specific sequences or simultaneously in laminar-flow configuration and was found to be suitable for delivering four classes of reagents to the devices: Small molecules, enzymes, antibodies and DNA. PNIPAM was also able to successfully deliver a series of standard glucose solutions to microPADs equipped to perform a colorimetric glucose assay. The results of these tests were used to produce an external calibration curve, which in turn was used to determine the concentration of glucose in sample solutions. Finally, PNIPAM was used to store the enzyme horseradish peroxidase for 35 days under ambient conditions with no significant loss of activity. The combination of PNIPAM and microPADs may allow for more complex assays to be performed on paper-based devices, facilitate the preparation of external calibration curves in the field, and extend the shelf life of microPADs by stabilizing reagents in an easy-to-use format.
\end{abstract}

Keywords: paper; microfluidics; PNIPAM; microPAD

\section{Introduction}

Paper-based fluidic devices (microPADs) have been developed over the past eight years as a new platform for simple, portable and low cost diagnostic tests [1,2]. These devices have the potential to 
allow for the qualitative and quantitative analysis of a variety of analytes - pathogens, toxins, hazardous chemicals, or biomarkers - in resource-limited settings such as remote areas in developed and developing countries. MicroPADs share many common features with other types of paper-based diagnostic devices such as dipstick assays and lateral-flow assays in that the devices are inexpensive, easy to use, and the results of the assays are usually reported as color changes [2,3]. What sets microPADs apart from other types of paper-based devices is that they incorporate networks of channels patterned into one or multiple pieces of paper that can transport fluids via capillary wicking [3]. These networks of channels can be used to perform multiple assays simultaneously or complex fluid handling steps so that more complex assays involving multiple steps could be performed on microPADs while still maintaining simplicity and low cost for the end user [2]. Some of the challenges faced by microPADs as a diagnostic platform are that multiple pipetting steps of sample and reagent solutions are still required for multistep assays, reagents are often not stable for extended periods of time when stored in dry form on the devices under ambient conditions, and freshly prepared external calibration curves are still required for quantitative analysis of the results, which means calibration standard solutions would have to be transported into the field so that external calibration assays could be conducted sideby-side with any test [4-8]. In an effort to overcome some of these limitations, we developed a convenient system for storing and then delivering reagent solutions to microPADs using the thermallyresponsive hydrogel $N, N^{\prime}$-methylenebisacrylamide-cross-linked poly(N-isopropylacrylamide) (PNIPAM).

Gels are unique polymer systems that are formed by cross-linking polymer chains to create a single large molecule [9]. Gels are classified into three major types depending on their physical properties: hydrogels, organogels and xerogels $[9,10]$. As the name suggests, hydrogels are compatible with aqueous solutions, which makes these gels particularly attractive for work with microPADs. We chose to focus on a specific class of hydrogels called thermally-responsive hydrogels. These gels incur conformational changes when subjected to temperature changes, which can be used to tune the water content of the gels [9-12]. PNIPAM is a hydrogel with a lower critical solution temperature (LCST) meaning this gel will collapse and expel aqueous solutions from its matrix when heated above its LCST [11-13]. This process is reversible, so a solution can be expelled from a PNIPAM gel at high temperatures, and the gel can then be loaded with a new solution by immersing the gel in the solution at low temperatures. In this way, PNIPAM gels can be used to store and then deliver reagent solutions to microPADs in response to temperature changes.

Hydrogels have long been associated with microfluidic devices. Microfluidic devices have been used to synthesize hydrogels, microfluidic devices have been fabricated out of hydrogels, and hydrogels have been incorporated into microfluidic devices to form valves, to form scaffolds for cell culture, and to trap or deliver analytes [14-20]. An article published recently by Niedl and Beta in Lab on a Chip introduced the use of hydrogels as fluid reservoirs for use with microPADs [21]. We had been developing the same concept concurrently without any knowledge of Niedl and Beta's work. While the work presented here and the work by Niedl and Beta both focus on the same general idea of combining hydrogels with microPADs, we have demonstrated different capabilities and applications, and, in many ways, the two articles complement each other. The article by Niedl and Beta focuses primarily on the use of hydrogels to deliver water to microPADs in order to drive sequential reactions with the reagents being pre-dried on the device. Our work involved a more detailed study of the use of hydrogels for storing and then delivering reagents in solution to microPADs. 
The experimental plan guiding this work had four primary objectives: (i) optimization of the PNIPAM gels for use with microPADs, (ii) characterization of the delivery of aqueous solutions from PNIPAM to microPADs, (iii) characterization of the delivery of reagents from PNIPAM to microPADs, and (iv) characterization of the storage of reagents, specifically enzymes, in PNIPAM for extended periods of time under ambient conditions. In terms of optimizing the PNIPAM gels for use with microPADs, we looked to synthesize gels that were durable, could be handled easily and could also be fabricated with reproducible dimensions. The cross-linker to monomer ratio was varied to produce a gel with the desired mechanical properties, and different types of molds were explored to control the dimensions of the gels. To characterize the delivery of aqueous solutions from PNIPAM gels to microPADs, we explored the delivery of a single solution to a device and also the delivery of multiple solutions either simultaneously or in a sequence to a device. Once the fluid-delivery of PNIPAM to microPADs was characterized, the ability of PNIPAM to deliver reagents to microPADs was assessed. This work focused on four major classes of reagents relevant to point-of-care diagnostic assays: water-soluble small molecules, enzymes, antibodies and DNA. There were three primary goals for this portion of the project: (i) to determine if PNIPAM was able to deliver the reagents to microPADs, (ii) to determine if PNIPAM had any effect on the concentration of the reagent it was delivering by either retaining or excluding it from the gel matrix, and (iii) to determine if PNIPAM could deliver the reagent accurately and precisely enough to construct an external calibration curve for a colorimetric assay. Finally, PNIPAM was assessed as a storage system for enzymes by measuring the activity of the enzyme horseradish peroxidase after being stored in PNIPAM gels for extended periods of time.

\section{Experimental Section}

\subsection{Reagents and Materials}

All reagents were purchased from commercial sources unless otherwise stated. The following chemicals were used: $N$-isopropylacrylamide (NIPAM, Sigma Aldrich), $N, N$ '-methylenebisacrylamide (BIS, Fisher Bioreagents), potassium persulfate (KPS, Sigma Aldrich), tetramethylethylenediamine (TEMED, Fisher Bioreagents), food coloring (Durkee), glucose (Sigma Aldrich), horseradish peroxidase (HRP, MP Biomedical), glucose oxidase (GOX, MP Biomedical), fluorescein tagged DNA (a 20 base pair-long oligonucleotide, Biosearch Technologies), fluorescein tagged rabbit anti-sheep IgG (Thermo Scientific), 2,2'-azino-bis(3-ethylbenzothioazoline-6-sulfonic acid) diammonium salt (ABTS, Alfa Aesar), and 1-Step ${ }^{\mathrm{TM}}$ ABTS (a proprietary solution of ABTS and $\mathrm{H}_{2} \mathrm{O}_{2}$, Thermo Scientific). A phosphate buffered saline solution (1XPBS) was prepared from 10XPBS (Fisher Scientific) with in-house nanopure water obtained from a nanopure dispenser (Thermo Scientific D13661). The following materials were obtained from commercially available sources and were used in the experiments: chromatography paper (Whatman No. 1), thermal laminating pouches (3 M, Scotch), and poly(ethylene) (PE) straws of three diameters: $11.03 \mathrm{~mm}$ (Karat), $7.24 \mathrm{~mm}$ (Crystal Ware), and $5.35 \mathrm{~mm}$ (Starbucks Coffee Company). 


\subsection{Fabrication of MicroPADs}

MicroPADs were fabricated by the method of wax patterning [22]. The patterns for the microPADs were designed in AutoCAD and then printed onto chromatography paper using a solid-ink printer (Xerox Phaser 8560). The paper was then baked in a convection oven (MTI Compact Forced Air) for 15 min at $145^{\circ} \mathrm{C}$ and then cooled to room temperature under ambient conditions. The devices were stored in plastic petri dishes until they were used.

After patterning the channels, some devices were enclosed with lamination sheets. Enclosing the devices was advantageous because it protected the channels from contamination and minimized evaporation of fluids from the channels. Hot lamination was performed by inserting the microPAD in a thermal laminating pouch and running the pouch through a thermal laminator (APACHE AL-12P, set to the $250^{\circ} \mathrm{F}$ setting).

\subsection{Digital Image Colorimetry}

The results from colorimetric assays conducted on microPADs were quantified via digital image colorimetry (DIC) $[7,23,24]$. The devices were first scanned using a desktop scanner (Epson Perfection V300) using the following settings: 48-bit Color image type, 300 dpi resolution, and reflective document type. Images were analyzed using ImageJ 1.46r, open source analysis software. The images were split into three color-channels: red, green and blue. The green and blue channels were discarded. The red channel was inverted, and the color intensity of the test zones was measured using a microarray profile plugin [25]. The mean intensity values obtained were analyzed in Excel.

\subsection{Synthesis of PNIPAM}

Poly( $N$-isopropylacrylamide) (PNIPAM) was synthesized via free radical solution polymerization. The synthesis was performed at room temperature using three purged vials. Molar ratios of monomer (NIPAM) to cross-linker (BIS) of 1:1, 3:1, 4:1, 5:1, 6:1, 7:1, 8:1, and 10:1 were tested. The following procedure is described for a monomer to cross-linker molar ratio of 5:1. Vial 1 contained NIPAM (1.209 $\mathrm{g}$, 10.68 mmoles $)$ and BIS (0.3294 g, 2.14 mmoles $)$ in $\mathrm{DI} \mathrm{H}_{2} \mathrm{O}(15 \mathrm{~mL})$. Vial 2 contained the KPS $(0.009 \mathrm{~g}$, 0.03 mmoles), a radical initiator, in DI $\mathrm{H}_{2} \mathrm{O}(2.0 \mathrm{~mL})$. Vial 3 contained TEMED $(71 \mu \mathrm{L}, 0.06 \mathrm{~g}$, 0.47 mmoles), a radical generator accelerator, in $\mathrm{DI}_{2} \mathrm{O}(2.0 \mathrm{~mL})$. Each vial was capped with a rubber septum, which was fastened with copper wire and then purged with nitrogen gas $\left(\mathrm{N}_{2}\right)$ for 20 min under atmospheric pressure. After purging, the contents of Vials 2 and 3 were added to Vial 1. Vial 1 was then taken off the nitrogen purge line, shaken thoroughly and allowed to react to completion at room temperature for $24 \mathrm{~h}$. The polymerization was considered to be complete when an opaque gel had solidified in Vial 1. This occurred typically within 10 min of combining the contents of the three vials, but the gel was left over night to ensure that the polymerization had gone to completion.

In order to create samples of PNIPAM with a defined surface area, PE straws with diameters of $5.37 \mathrm{~mm}, 7.24 \mathrm{~mm}$, and $11.03 \mathrm{~mm}$ were used as inexpensive molds for PNIPAM. The straws were placed into Vial 1 prior to purging and retrieved with molded PNIPAM once the reaction was complete. To produce varying lengths of sample, the length of the straws were varied as well as the size of the vial. 
Regardless of the size of the vial, molar ratios of the reagents were kept constant (i.e., NIPAM (5): BIS (1): TEMED (0.22): KPS (0.016)) and solvent volumes were scaled proportionally.

After $24 \mathrm{~h}$, the reaction vial (Vial 1) was etched with a glass cutter above the level of the gel. The rubber septum was then removed, and the vial was immersed in a liquid nitrogen bath for approximately $45 \mathrm{~s}$, ensuring that the entire etched path was exposed to liquid nitrogen. Immediately after liquid nitrogen exposure, the vial was shattered along the etch line using channel-lock pliers. The vial was covered with an aluminum foil sheet to contain the shattered glass. The straw-encased PNIPAM hydrogel samples were then retrieved from the PNIPAM mass.

The hydrogels were thermocycled to purify the polymer matrix by removing any residual reagents (Figure $\mathrm{S} 1$ ). The thermocycling procedure involved the following steps: (i) the PNIPAM was submerged in a vial containing DI $\mathrm{H}_{2} \mathrm{O}$ and heated for 10 minutes at $60^{\circ} \mathrm{C}$ in a silicon oil bath, (ii) the water was decanted and the samples were rinsed in the vial with fresh portions of $\mathrm{DI}_{2} \mathrm{O}$ two times, (iii) the vial was filled with fresh $\mathrm{DI} \mathrm{H}_{2} \mathrm{O}$ and placed in an ice-water bath $\left(0^{\circ} \mathrm{C}\right)$ for $20 \mathrm{~min}$, (iv) the vial was removed from the ice-water bath and allowed to warm to room temperature. The cycle was repeated a total of 6 times for each hydrogel.

\subsection{Characterization of Fluid Delivery from PNIPAM}

To determine the amount of fluid that could be released by PNIPAM upon heating, we heated samples of PNIPAM of each of the three diameters for $12 \mathrm{~min}$ on a digital hotplate set to $40^{\circ} \mathrm{C}$ and recorded the initial and final mass of the samples. The duration of the heating step (12 min) was an arbitrary amount of time selected because we believe it is a reasonable time scale for the operation of microPADs in the real world. The temperature of $40{ }^{\circ} \mathrm{C}$ was chosen because it is achievable using a HotHands ${ }^{\circledR}$ 's hand warmer, which is an inexpensive, commercially available and portable heat source, and it is conceivable that a health professional could use this type of product in the field to perform a paper-based diagnostic assay with PNIPAM as a fluid source. Several other instrument-free approaches for controlling temperature of paper microfluidic devices have also been described and could be used with PNIPAM [26]. Using the same approach, we also studied the effect of heating time at $40{ }^{\circ} \mathrm{C}$ on the amount of fluid expelled by PNIPAM gels.

\subsection{Loading Reagents into PNIPAM}

To load reagents or dyes into the hydrogel, the gel was first heated to expel all fluid, then transferred to a vial with the desired reagent solution and allowed to equilibrate at room temperature for $24 \mathrm{~h}$. The gel was heated by submerging it in $\mathrm{DI}_{2} \mathrm{O}$ in a glass vial, and then placing the vial into a silicone oil bath set to $60{ }^{\circ} \mathrm{C}$. The temperature of the oil bath was increased by $10{ }^{\circ} \mathrm{C}$ every $30 \mathrm{~min}$ to a final temperature of $80^{\circ} \mathrm{C}$, and the final temperature was held for $30 \mathrm{~min}$.

\subsection{Delivery of Fluids from PNIPAM to MicroPADs}

PNIPAM gels (7.24-mm in diameter and 1-cm in length) were loaded with aqueous dye solutions and placed on microPADs to study fluid delivery to paper. One microPAD was used to measure the rate of wicking for fluids delivered to paper-based devices via PNIPAM at room temperature and at $40{ }^{\circ} \mathrm{C}$. This 
device consisted of a single circular sample addition zone with a diameter of $7.3 \mathrm{~mm}$ leading into a 6-cm-long and 2-mm-wide channel with markings every centimeter along both sides of the channel. For comparison, the same experiment was carried out by adding $45 \mu \mathrm{L}$ of solution in three $15-\mu \mathrm{L}$ aliquots directly to the sample inlet using a micropipette. A second microPAD was used to demonstrate that PNIPAM could deliver fluids in laminar flow. This device had three circular sample addition zones all with a diameter of $7.3 \mathrm{~mm}$ that were situated at one end of the device and were equidistant from the point of entry into a single channel that led to a waste zone. A third microPAD was used to demonstrate that PNIPAM could deliver fluids to a reaction zone in a specific sequence. This device had three staggered circular sample addition zones all with a diameter of $7.3 \mathrm{~mm}$ leading into a single channel that emptied into a waste zone. All three devices were laminated, and the lamination over the sample addition and waste zones was cut out and removed using a razor blade to allow for sample introduction and for fluids to evaporate from the waste zone. For the second and third devices, PNIPAM gels, loaded with red dye, blue dye and water respectively, were placed in the three sample addition zones at the same time, and the device was heated to $40{ }^{\circ} \mathrm{C}$ to observe the delivery of fluid from the gels to the devices.

\subsection{Delivery of Reagents from PNIPAM to MicroPADs}

PNIPAM was used to deliver four distinct, clinically-relevant classes of analytes to microPADs: small molecules (glucose), enzymes (HRP), antibodies (fluorescein-labeled IgG) and DNA (fluoresceinlabeled 20 base pair oligonucleotide).

A paper-based 96-well plate with 5-mm-diameter circular reaction zones was used to demonstrate the delivery of glucose from a PNIPAM gel to a microPAD [27]. The PNIPAM gels used for these experiments had a diameter of $7.24 \mathrm{~mm}$ and length of approximately $4 \mathrm{~cm}$. The concentration of glucose delivered to the reaction zones was quantified using a colorimetric glucose assay based on a coupled enzymatic reaction using GOX, HRP, and ABTS, which produced a blue-green color in the reaction zones.

Glucose solutions of the following concentrations were prepared in duplicate: $0 \mathrm{mM}\left(\mathrm{DI} \mathrm{H}_{2} \mathrm{O}\right), 1 \mathrm{mM}$, $2 \mathrm{mM}, 2.5 \mathrm{mM}, 3 \mathrm{mM}, 3.5 \mathrm{mM}, 4 \mathrm{mM}$, and $5 \mathrm{mM}$. One set of the glucose solutions was loaded into PNIPAM gels. The other set was used as external solutions for comparison. Ultimately, three different types of glucose samples were prepared: glucose loaded into PNIPAM (PNIPAM), the excess glucose solutions remaining in the vials after loading the PNIPAM gels (PNIPAM solution), and the external glucose solutions (solution). A reaction mixture was prepared consisting of GOX $\left(75 \mathrm{kU} \cdot \mathrm{L}^{-1}\right)$, HRP $\left(250 \mathrm{kU} \cdot \mathrm{L}^{-1}\right)$ and ABTS $(6.25 \mathrm{mM})$ in 1 XPBS. A volume of $1 \mu \mathrm{L}$ of reaction mixture was spotted in the reactions zones of a paper-based 96-well plate and dried for $30 \mathrm{~min}$ under ambient conditions. The PNIPAM samples were placed in the wells for $60 \mathrm{~s}$ and then removed. For the PNIPAM loading solution and the external glucose solutions, $3 \mu \mathrm{L}$ of each solution was added to the reaction zone using a micropipette. Three replicates were performed for each concentration. The colorimetric assays were allowed to develop for $30 \mathrm{~min}$ before being imaged and analyzed. Two calibration standard glucose solutions with concentrations of $2.5 \mathrm{mM}$ and $3.5 \mathrm{mM}$, respectively, were also tested using the same colorimetric assay to evaluate the accuracy and precision of the assay.

To evaluate the delivery of fluorescein tagged antibody and DNA, devices with a circular sample addition zone and a circular test zone, both with a diameter of $7.25 \mathrm{~mm}$, connected by a single channel (length $2 \mathrm{~mm}$ and width $2.2 \mathrm{~mm}$ ) were used. Two sets of solutions with concentrations of $750 \mathrm{mg} / \mathrm{L}$ and 
$93.8 \mathrm{mg} / \mathrm{L}$ of fluorescein-labeled rabbit anti-sheep IgG were prepared in 1XPBS. One set of the solutions was loaded into 7.24-mm PNIPAM gels with an average mass of $0.1504 \mathrm{~g}$, and the other set of solutions was used as external solutions for comparison. The PNIPAM gels were placed upon the sample addition zone of the microPADs at room temperature until the solution completely filled the test zone. The external solutions were tested by adding $10 \mu \mathrm{L}$ of solution to the sample addition zone. All devices were dried for $20 \mathrm{~min}$. Once the devices were dry, they were imaged using a fluorescent scanner (Typhoon TRIO+, Amersham Biosciences) and quantified in ImageJ.

Two sets of solutions of fluorescein tagged DNA (20 base-pair-long oligonucleotide) with concentrations of $7.95 \mu \mathrm{M}$ and $0.994 \mu \mathrm{M}$ were prepared in 1XPBS. One set of solutions was loaded into 7.24-mm PNIPAM gels with an average mass of $0.1389 \mathrm{~g}$, and the other set of solutions was used as external solutions for comparison. Samples were tested by the same method as the fluorescein-labeled IgG samples.

\subsection{Storage of Reagents in PNIPAM}

PNIPAM was assessed for its ability to deliver an enzyme, HRP, by passive wicking to a microPAD using a paper-based 96-well plate. The effects of PNIPAM on the activity of HRP were also investigated. PNIPAM samples with a 7.24-mm diameter and length of approximately $4 \mathrm{~cm}$ were prepared for loading with HRP solution. A working HRP solution was prepared with a concentration of $550 \mathrm{kU} \cdot \mathrm{L}^{-1}$ in 1XPBS. A portion of this solution was loaded into eight PNIPAM gels. PNIPAM gels loaded with 1XPBS were used as negative controls to account for any background signal from the assay. The remaining HRP solution was used as a control solution. After the PNIPAM gels were loaded with the HRP solution, they were divided into two groups: four gels were stored in dry, empty vials (dry storage), and the other four gels were stored in the HRP solution (solution storage). The dry storage gels, solution storage gels, and the HRP solution were all prepared on the same day (day 0 ) and then stored in a drawer at room temperature for the duration of the experiment. The activity of the HPR stored under the three conditions was monitored on select days up to day 35 .

The activity of the HRP in the three samples was monitored using a colorimetric assay with ABTS. The PNIPAM samples (3-mm sections of the gels) were placed on reaction zones of a paper-based 96-well plate for $10 \mathrm{~min}$ to ensure delivery of HRP. For comparison, $1 \mu \mathrm{L}$ of the HRP solution was added to separate reaction zones. Immediately after adding the HRP solutions, $3 \mu \mathrm{L}$ of 1 Step ABTS was added to each reaction zone. The color from the assay was allowed to develop for 30 min under ambient conditions, and then the results were imaged and quantified.

As an additional experiment we assessed if a single PNIPAM gel could deliver reproducible amounts of HRP in sequential trials. The PNIPAM samples were placed in a reaction zone for 10 min for each trial. After $10 \mathrm{~min}$, the samples were transferred directly to a new reaction zone. This process was repeated four times. The activity of the HRP delivered to the test zones was determined using the ABTS assay. 


\section{Results and Discussion}

\subsection{Synthesis and Characterization of PNIPAM}

The synthesis of PNIPAM was optimized to produce a hydrogel that could be handled easily (Figure 1). It was determined that a monomer to cross-linker molar ratio of 5:1 was optimal for producing PNIPAM that was easy to handle and cut. Gels synthesized with ratios of monomer to cross-linker greater than 5:1 were too soft to handle effectively, while the gels with a lower ratio were too brittle. The ability to handle the material was crucial in this project, because it allowed for the gels to be cut into discrete masses efficiently, and for the segments to be manipulated while maintaining their shape and size. The monomer to cross-linker ratio of 5:1 was used throughout the work described in this article.

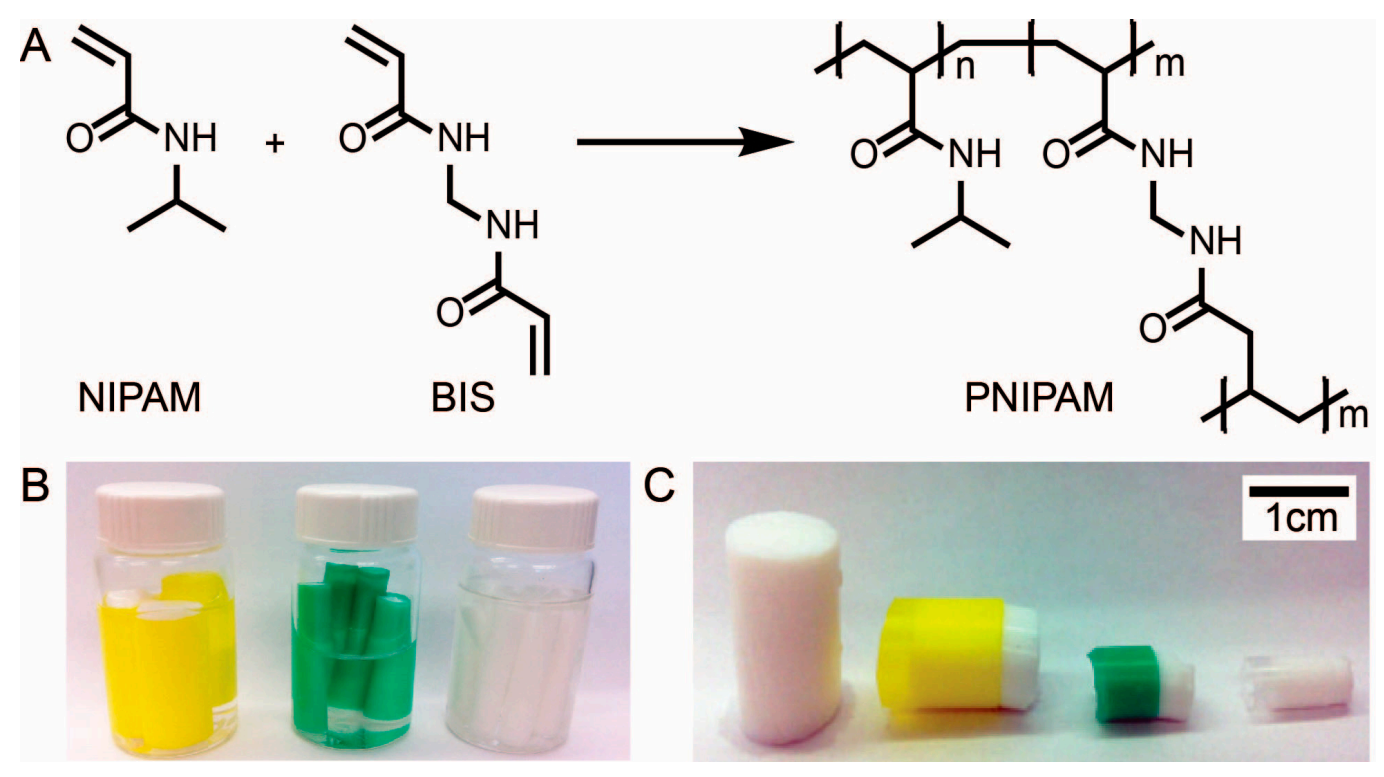

Figure 1. (a) Synthesis of PNIPAM from NIPAM monomer and BIS cross-linker. (b) Three reaction vials containing cylindrical PNIPAM gels in the three diameters of $11.03 \mathrm{~mm}$, $7.24 \mathrm{~mm}$, and $5.35 \mathrm{~mm}$, respectively, stored in DI $\mathrm{H}_{2} \mathrm{O}$. (c) An unsheathed PNIPAM sample with a diameter of $11.03 \mathrm{~mm}$ followed by sheathed samples of PNIPAM with diameters of $11.03 \mathrm{~mm}, 7.24 \mathrm{~mm}$, and $5.35 \mathrm{~mm}$, respectively.

The use of straws proved to be an effective method of molding PNIPAM into cylinders of reproducible diameters (Figure 1b,c). We chose to work with straws that were commercially available, but this method of synthesis could presumably be used to prepare PNIPAM samples in a variety of shapes as long as the appropriate plastic casing was available.

The mass of fluid delivered by PNIPAM gels was found to be strongly dependent on the surface area of the PNIPAM exposed to the heat source (Figure S2). For PNIPAM samples of the same initial mass, the sample with the largest diameter - and therefore the largest surface area in contact with the heat source - consistently expelled the most fluid. We also observed that the amount of fluid delivered eventually reached a plateau as the initial mass of the sample was increased. This result suggests that the amount of fluid delivered by a gel is limited by the area of the gel exposed to the heat source. Fortunately, most microPADs require only small volumes $(5-50 \mu \mathrm{L})$ of fluid to operate, and these volumes can be delivered using the 11.03-mm and 7.24-mm diameter PNIPAM gels. Longer heating times were also 
shown to increase the amount of fluid expelled from the PNIPAM gels (Figure S3). The results of these characterization experiments could be useful for predicting the mass of fluid that could be delivered by a given PNIPAM sample when heated to $40{ }^{\circ} \mathrm{C}$. When delivering fluids to microPADs, it is important that the PNIPAM gel be able to deliver an excess of fluid compared to the amount of fluid required to fill the particular device. This way the volume of fluid delivered to the device will be controlled by the dimensions of the device and not by the PNIPAM gel, and thus the volume of fluid delivered to the device will be reproducible from one experiment to the next [5].

\subsection{Delivery of Fluids from PNIPAM to MicroPADs}

We found that PNIPAM can effectively deliver fluids to microPADs via a traditional circular sample inlet zone, but with a slightly different wicking profile compared to when solution is delivered directly to the microPAD's inlet zone (Figure 2a,b). An interesting and unexpected observation was that the PNIPAM gels delivered fluid to the microPAD even at room temperature, which was below the LCST of the polymer. The gels were only observed to expel fluid at room temperature when in contact with paper. Fluid delivery from the gels to paper at room temperature is suspected to occur due to the fluid interacting favorably with paper and thus being wicked out of the PNIPAM matrix. When the PNIPAM sample was heated to $40{ }^{\circ} \mathrm{C}$, fluid was delivered more rapidly to the device, and the wicking rate increased compared to room temperature conditions. These results suggest that PNIPAM and temperature could be used to tune the wicking rate and total mass of fluid delivered to a microPAD, which could be useful for controlling the movement of fluids in a device. This temperature control over wicking rate may allow for fluids to be delivered in a specific sequence from various PNIPAM samples on the same device by selectively heating the gels.

Once the basic delivery of fluids from PNIPAM to microPADs was demonstrated, we demonstrated that PNIPAM could deliver fluids in two classic microfluidic configurations: laminar flow and plugreagent delivery (Figure 2c,d). We see these experiments as a first step toward developing higher-level functionality of microPADs with PNIPAM. The device shown in Figure $2 \mathrm{c}$ demonstrates that PNIPAM can deliver fluids simultaneously from neighboring sample zones into a single channel and that the fluids wick across the channel in laminar flow. A gradient of both blue and red dye may be seen across the width of the channel after $60 \mathrm{~min}$. This result suggests that the simultaneous addition of fluids could be used to produce gradients of reagents in microPADs. The device shown in Figure $2 \mathrm{~d}$ demonstrates PNIPAM's ability to add fluids to a microPAD as plugs with minimal mixing and in a defined sequence. This sequential addition of reagents could be useful for performing a multi-step assay such as an ELISA [28].

\subsection{Delivery of Reagents from PNIPAM to MicroPADs}

PNIPAM was used successfully to store and then deliver glucose, fluorescein-labeled rabbit antisheep IgG, fluorescein-labeled DNA, and HRP to microPADs. These reagents are representative of four major classes of analytes that are interesting for diagnostic purposes: small molecules, antibodies, DNA, and enzymes. 

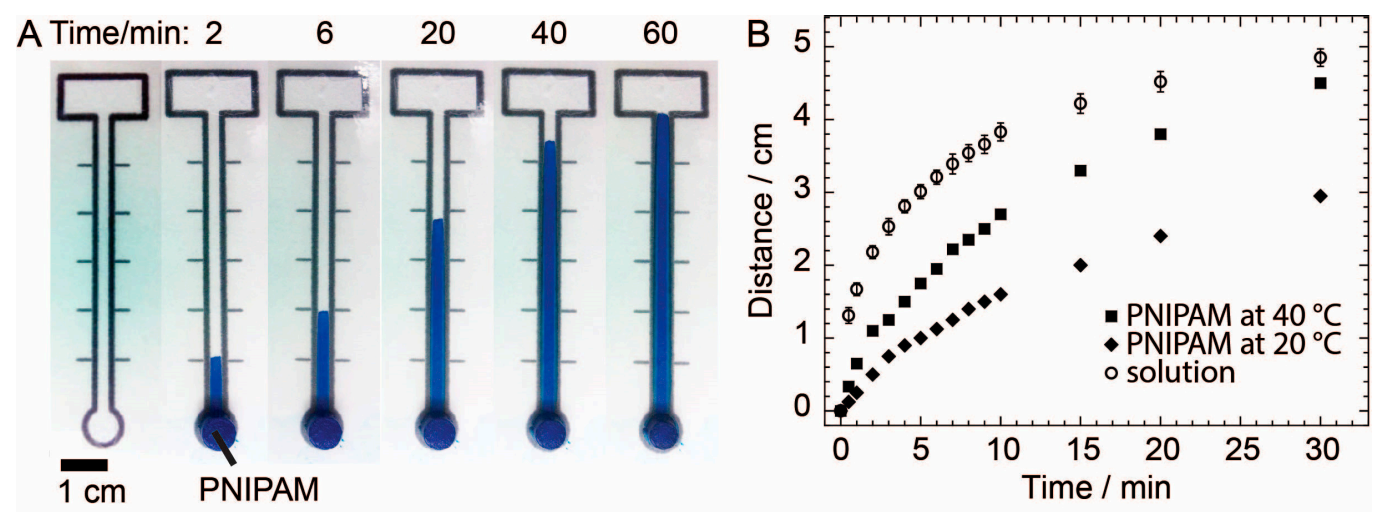

C Time/min: 0
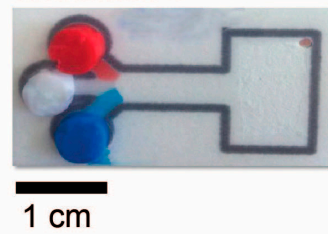

D Time/min: 0

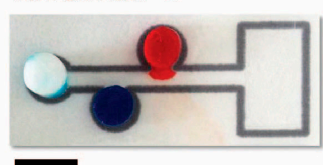

$\overline{1 \mathrm{~cm}}$
3

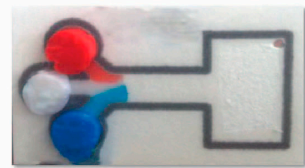

10

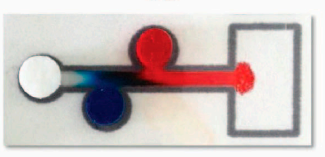

40

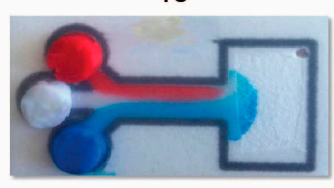

30

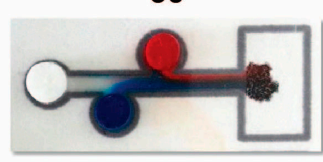

60

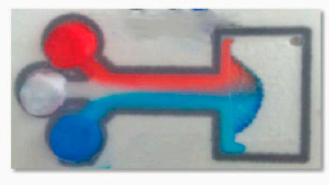

130

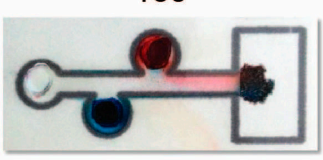

Figure 2. (a) A sequence of images documenting the delivery of an aqueous blue dye solution from a PNIPAM gel to a microPAD heated to $40{ }^{\circ} \mathrm{C}$. (b) A plot of the distance wicked by fluid in the microPAD shown in (a) versus time for fluids delivered by PNIPAM gels either at $40{ }^{\circ} \mathrm{C}$ or room temperature $\left(20{ }^{\circ} \mathrm{C}\right)$ or delivered by micropipette. Data points represent the mean of three trials, and error bars represent one standard deviation from the mean. (c) A sequence of images documenting the simultaneous delivery of three solutions from PNIPAM gels to a microPAD. The fluids participate in laminar flow. (d) A sequence of images documenting the delivery of three solutions from PNIPAM gels to a microPAD in distinct plugs. Dye diffusion is minimal between the different solutions.

The results for the delivery of glucose from PNIPAM gels to microPADs are shown in Figure 3. These results demonstrate that PNIPAM can deliver glucose to microPADs in a concentration-dependent fashion, that loading glucose into PNIPAM has no significant effect on the concentration of glucose in the loading solution, and that delivering glucose to microPADs from PNIPAM has no significant effect on the concentration of glucose in solution. The results indicate that glucose is not retained by or excluded from the PNIPAM matrix when it is loaded into the gel. This is promising first evidence that small molecules, at least of similar polarity to glucose, could be loaded into PNIPAM and delivered to microPADs without any effect on their concentration. The results also suggest that PNIPAM samples loaded with standard solutions could be taken into the field and used to prepare external calibration curves at the point-of-care. 


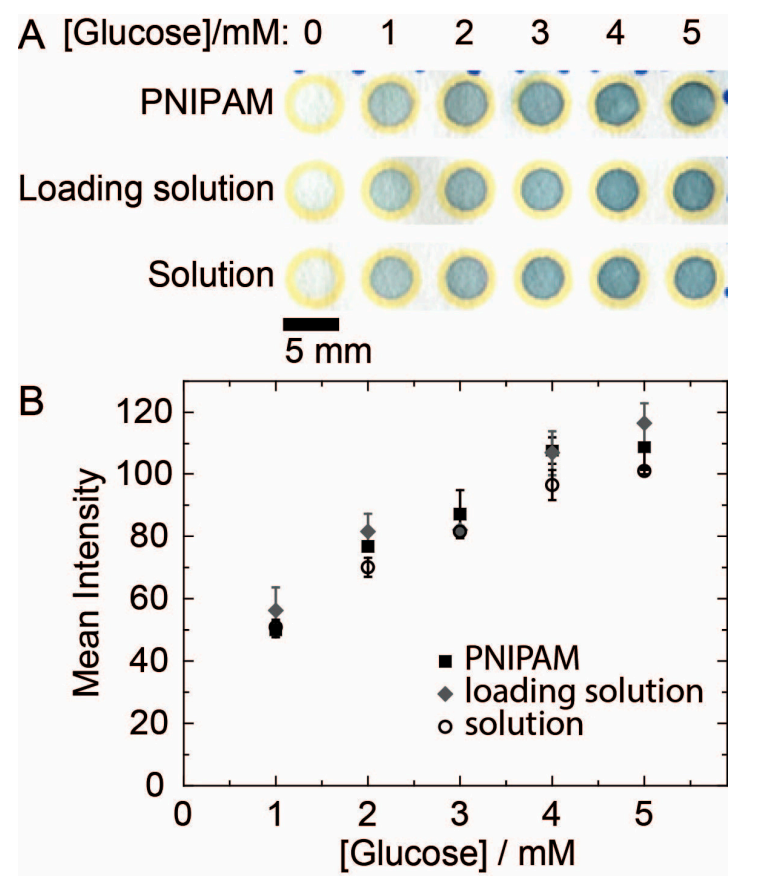

Figure 3. (a) Scanned image of the results of glucose assays performed in paper-based test zones. The intensity of the color produced in the test zone increases as the concentration of glucose increases. The glucose solution was delivered to the test zone either from a PNIPAM gel or as a solution using a micropipette. (b) Plot of color intensity versus concentration of glucose. Data points represent the mean of three measurements, and error bars represent one standard deviation from the mean.

The results from the PNIPAM-delivered glucose solutions were fit with a linear trendline and used as a calibration curve to determine the concentrations of glucose in two calibration standard solutions delivered both from a PNIPAM gel and by pipette (Table 1). The determined concentrations of all four glucose samples were found to be within one standard deviation of their true concentrations. The relative standard deviations of the measurements were all within 15\%. The accuracy and precision of these results is comparable to the results obtained typically for microPADs using pipet-delivered solutions [7]. These results support the idea that PNIPAM gels loaded with external standard solutions could be used for preparing external calibration curves with microPADs, and also suggest that samples could be collected in the field and stored for later testing by loading them into PNIPAM gels.

Table 1. Results for the determination of the concentration of glucose in calibration standard solutions. Results are reported as the mean of three measurements, and uncertainties are reported as one standard deviation from the mean.

\begin{tabular}{ccc}
\hline $\begin{array}{c}\text { Concentration of Glucose } \\
\text { in Calibration Standard }\end{array}$ & $\begin{array}{c}\text { Source of Calibration } \\
\text { Standard }\end{array}$ & $\begin{array}{c}\text { Determined Concentration } \\
\text { of Glucose }\end{array}$ \\
\hline \multirow{2}{*}{$2.5 \mathrm{mM}$} & PNIPAM & $2.6 \pm 0.5 \mathrm{mM}$ \\
& Pipette & $2.5 \pm 0.2 \mathrm{mM}$ \\
\multirow{2}{*}{$3.5 \mathrm{mM}$} & PNIPAM & $3.6 \pm 0.4 \mathrm{mM}$ \\
& Pipette & $3.4 \pm 0.5 \mathrm{mM}$ \\
\hline
\end{tabular}


PNIPAM was also shown to deliver a fluorescein-labeled antibody and fluorescein-labeled DNA to microPADs with concentration dependence and with no significant difference in signal compared to the same analytes delivered using micropipettes (Figure 4 and Table S1). These results suggest that antibodies and DNA could also be delivered to microPADs from PNIPAM gels to prepare external calibration curves or for testing samples in the field.
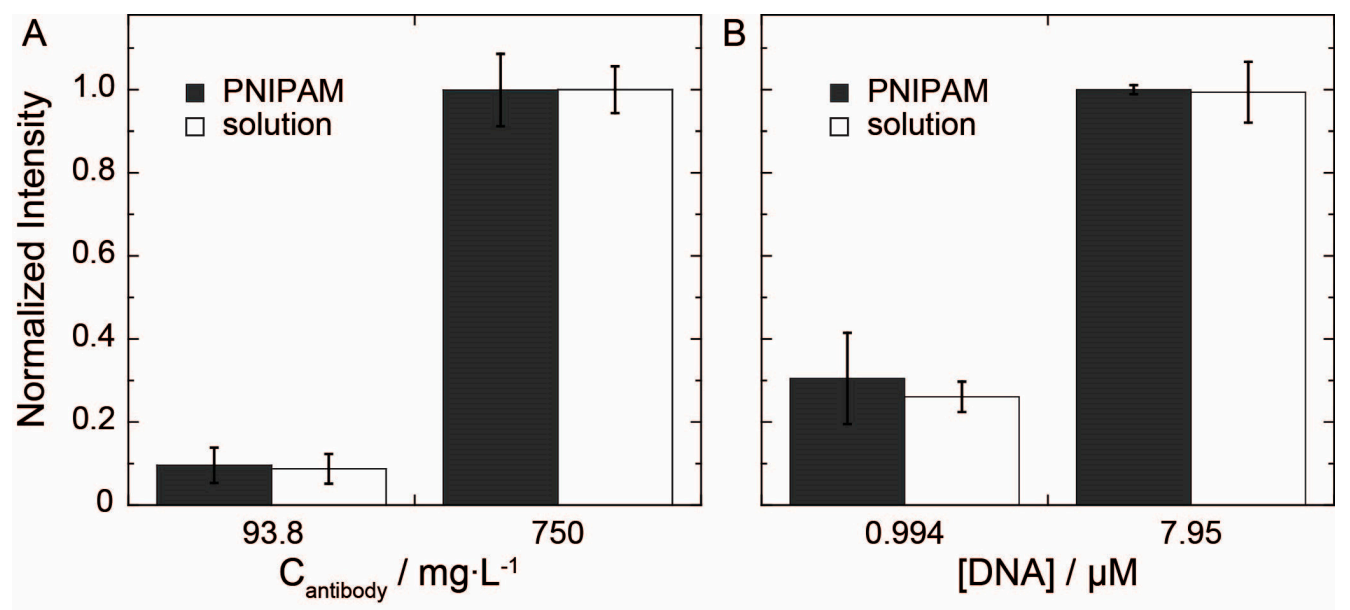

Figure 4. (a) Plot of the normalized fluorescence intensity versus concentration for PNIPAM-delivered fluorescein-labeled antibody (PNIPAM) and pipette-delivered fluorescein-labeled antibody (solution). (b) Plot of the normalized fluorescence intensity versus concentration for PNIPAM-delivered and pipette-delivered fluorescein-labeled DNA. The height of each bar represents the mean of three measurements, and the error bars represent one standard deviation from the mean.

\subsection{Storage of Reagents in PNIPAM}

PNIPAM was first shown to deliver HRP to paper via passive wicking (Figure 5). The appearance of the blue-green color in the test zones upon addition of the ABTS reagent solution confirmed that the enzyme was delivered to the paper test zone and was still active. Once the ability to deliver HRP was confirmed, the PNIPAM samples were reused three more times producing consistent signals. The signals from the PNIPAM-delivered samples were also consistently larger than the signals from the pipettedelivered samples, but this is probably because more HRP solution was delivered to the test zone from the PNIPAM gels. The results suggest that PNIPAM gels loaded with specific reagents could be used for several assays without any loss in effective concentration of the reagent delivered. An end-user could potentially work with one set of reagent-loaded PNIPAM gels for an extended period of time, reusing them for multiple assays.

Figure $5 \mathrm{~b}$ compares the activity of HRP for three different storage conditions as a function of time. The first storage condition was HRP in PNIPAM and stored in a dry vial (dry storage). The second was HRP in PNIPAM and stored in a vial full of HRP solution (solution storage). The third was HRP in solution (solution). The activity of HRP for all three conditions was found to be comparable on each day of the 35-day trial. These results indicate that PNIPAM has no effect on the stability of HRP, and that PNIPAM has the potential to be used as a storage matrix for sensitive biological reagents. HRP is only able to retain its activity for a few days when stored on paper in dry form [5,6]. PNIPAM keeps the 
enzyme stable for at least 35 days and probably longer considering that the signal for the assay had shown no significant decrease from the signal on day 0 . These results suggest that reagents for assays could be loaded into PNIPAM gels, transported into the field at room temperature, and then delivered to microPADs just prior to conducting an assay at the point-of-care.
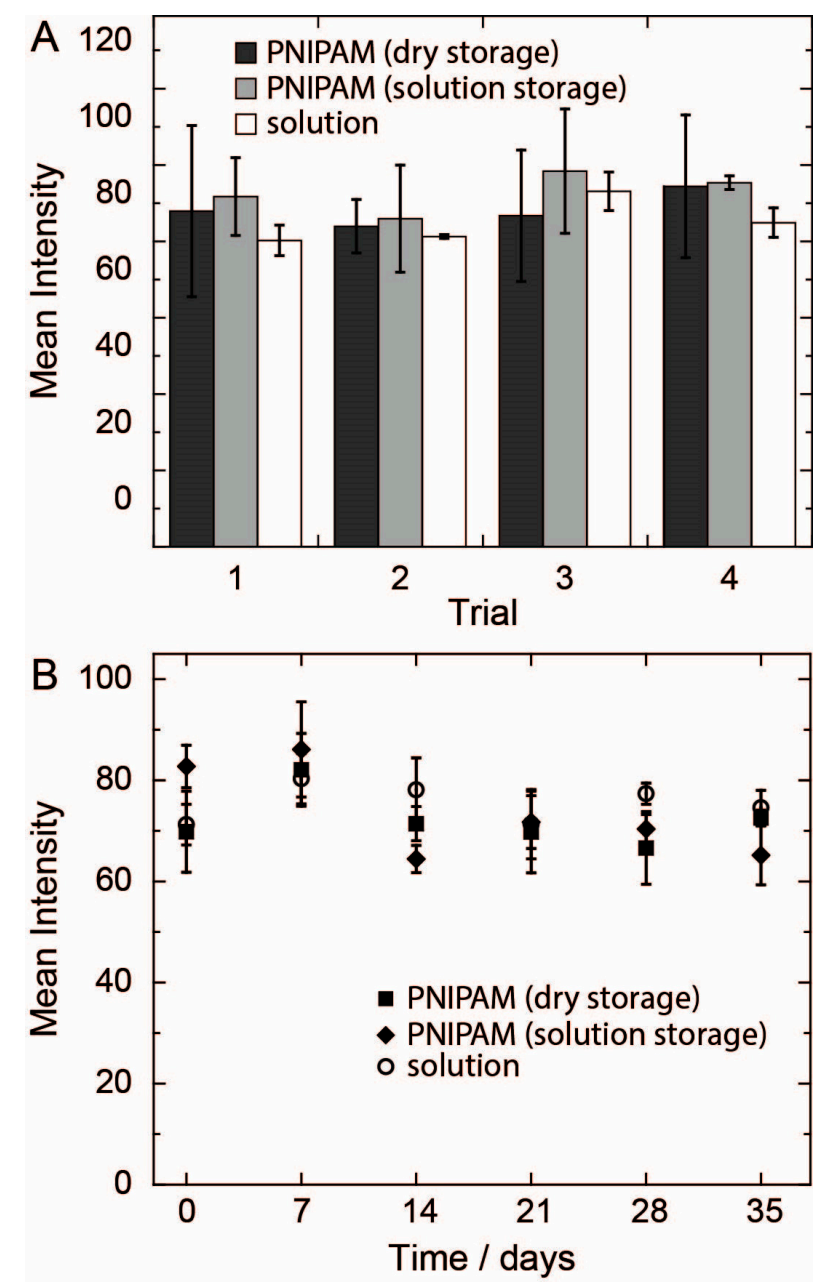

Figure 5. (a) Plot of the mean intensity of the colorimetric signal measured from the test zones versus the number of times each PNIPAM sample was used to deliver HRP to test zones. The height of each bar represents the mean of three measurements and error bars represent one standard deviation from the mean. (b) Plot of mean intensity of the colorimetric signal versus time for HRP stored under three different conditions. Data points represent the mean of three measurements, and error bars represent one standard deviation from the mean.

\section{Conclusions}

We introduced a straightforward method for synthesizing PNIPAM gels with reproducible dimensions and of the appropriate size for use with microPADs. PNIPAM was shown to be a promising fluid delivery system for use with microPADs. When combined with PNIPAM gels, microPADs may be capable of relatively complex fluidic operations with minimal input from the user. PNIPAM also showed great potential as a material for storing and delivering clinically relevant reagents to microPADs in a concentration dependent manner. Since PNIPAM provides a convenient platform for storing 
solutions of reagents, we believe PNIPAM gels loaded with reagents could be transported into the field and then used to produce external calibration curves at the point-of-care without the need for pipettes. Furthermore, the synthesis and processing of PNIPAM is simple, inexpensive, and scalable making it a plausible candidate for use in low-cost diagnostics for the developing world.

\section{Supplementary Materials}

Supplementary materials can be accessed at: http://www.mdpi.com/2227-9075/2/3/436/s1.

\section{Acknowledgments}

This project was supported by a Non-Tenured Faculty Grant from 3M, a seed grant from the Cal Poly Center for Applications in Biotechnology funded by the Chevron Biotechnology Applied Research Endowment, and a Research, Scholarly, and Creative Activities grant from California Polytechnic State University, San Luis Obispo. We would like to thank Ray Fernando and the Kenneth N. Edwards Western Coatings Technology Center at Cal Poly for allowing us to use their facilities for synthesis of PNIPAM, Kevin Dunham and Lisa Liu for help with obtaining supplies and equipment, and Starbucks for allowing us to take some of their green straws for our project.

\section{Author Contributions}

Haydn Mitchell, Philip Costanzo and Andres Martinez contributed to the design of the experiments and interpretation of the results. Haydn Mitchell performed all experimental work. Haydn Mitchell, Spencer Schultz, Philip Costanzo and Andres Martinez contributed to writing and revising the manuscript.

\section{Conflicts of Interest}

The authors declare no conflict of interest.

\section{References}

1. Martinez, A.W.; Phillips, S.T.; Butte, M.J.; Whitesides, G.M. Patterned paper as a platform for inexpensive, low-volume, portable bioassays. Angew. Chem. Int. Ed. 2007, 46, 1318-1320.

2. Yetisen, A.K.; Akram, M.S.; Lowe, C.R. Paper-based microfluidic point-of-care diagnostic devices. Lab Chip 2013, 13, 2210-2251.

3. Martinez, A.W.; Phillips, S.T.; Whitesides, G.M.; Carrilho, E. Diagnostics for the developing world: Microfluidic paper-based analytical devices. Anal. Chem. 2010, 82, 3-10.

4. Fu, E.; Kauffman, P.; Lutz, B.; Yager, P. Chemical signal amplification in two-dimensional paper networks. Sensor. Actuat. B Chem. 2010, 149, 325-328.

5. Martinez, A.W.; Phillips, S.T.; Carrilho, E.; Thomas, S.W.; Sindi, H.; Whitesides, G.M. Simple telemedicine for developing regions: Camera phones and paper-based microfluidic devices for realtime, off-site diagnosis. Anal. Chem. 2008, 80, 3699-3707.

6. Mitchell, H.T.; Noxon, I.C.; Chaplan, C.A.; Carlton, S.J.; Liu, C.H.; Ganaja, K.A.; Martinez, N.W.; Immoos, C.E.; Costanzo, P.J.; Martinez, A.W. Reagent pencils: A new technique for solvent-free deposition of reagents onto paper-based microfluidic devices. Lab Chip 2015, 17-20. 
7. Chaplan, C.A.; Mitchell, H.T.; Martinez, A.W. Paper-based standard addition assays. Anal. Methods 2014, 6, 1296.

8. Murdock, R.C.; Shen, L.; Griffin, D.K.; Kelley-Loughnane, N.; Papautsky, I.; Hagen, J.A. Optimization of a paper-based ELISA for a human performance biomarker. Anal. Chem. 2013, 85, 11634-11642.

9. Odian, G. Principles of Polymerization, 4th ed.; John Wildey \& Sons: Hoboken, NJ, USA, 2004.

10. Meng, H.; Hu, J. A Brief Review of Stimulus-active Polymers Responsive to Thermal, Light, Magnetic, Electric, and Water/Solvent Stimuli. J. Intell. Mater. Syst. Struct. 2010, 21, 859-885.

11. Ono, Y.; Shikata, T. Hydration and dynamic behavior of poly( $\mathrm{N}$-isopropylacrylamide)s in aqueous solution: A sharp phase transition at the lower critical solution temperature. J. Am. Chem. Soc. 2006, $128,10030-10031$.

12. Zhang, Y.; Furyk, S.; Bergbreiter, D.E.; Cremer, P.S. Specific ion effects on the water solubility of macromolecules: PNIPAM and the Hofmeister series. J. Am. Chem. Soc. 2005, 127, 14505-14510.

13. Tucker, A.K.; Stevens, M.J. Study of the Polymer Length Dependence of the Single Chain Transition Temperature in Syndiotactic Poly(N-isopropylacrylamide) Oligomers in Water. Macromolecules 2012, 45, 6697-6703.

14. Huber, D.L.; Manginell, R.P.; Samara, M.A.; Kim, B.-I.; Bunker, B.C. Programmed adsorption and release of proteins in a microfluidic device. Science 2003, 301, 352-354.

15. Annabi, N.; Selimović, Š.; Acevedo Cox, J.P.; Ribas, J.; Afshar Bakooshli, M.; Heintze, D.; Weiss, A.S.; Cropek, D.; Khademhosseini, A. Hydrogel-coated microfluidic channels for cardiomyocyte culture. Lab Chip 2013, 13, 3569-3577.

16. Cheng, S.-Y.; Heilman, S.; Wasserman, M.; Archer, S.; Shuler, M.L.; Wu, M. A hydrogel-based microfluidic device for the studies of directed cell migration. Lab Chip 2007, 7, 763-769.

17. Heo, J.; Crooks, R.M. Microfluidic biosensor based on an array of hydrogel-entrapped enzymes. Anal. Chem. 2005, 77, 6843-6851.

18. Hwang, D.K.; Dendukuri, D.; Doyle, P.S. Microfluidic-based synthesis of non-spherical magnetic hydrogel microparticles. Lab Chip 2008, 8, 1640-1647.

19. Song, Y.S. A passive microfluidic valve fabricated from a hydrogel filled with carbon nanotubes. Carbon NY 2012, 50, 1417-1421.

20. Kanai, T.; Ohtani, K.; Fukuyama, M.; Katakura, T.; Hayakawa, M. Preparation of monodisperse PNIPAM gel particles in a microfluidic device fabricated by stereolithography. Polym. J. 2011, 43, 987-990.

21. Niedl, R.R.; Beta, C. Hydrogel-driven paper-based microfluidics. Lab Chip 2015, 15, 2452-2459.

22. Carrilho, E.; Martinez, A.W.; Whitesides, G.M. Understanding wax printing: A simple micropatterning process for paper-based microfluidics. Anal. Chem. 2009, 81, 7091-7095.

23. Byrne, L.; Barker, J.; Pennarun-Thomas, G.; Diamond, D.; Edwards, S. Digital imaging as a detector for generic analytical measurements. TrAC - Trends Anal. Chem. 2000, 19, 517-522.

24. Lopez-Molinero, A.; Liñan, D.; Sipiera, D.; Falcon, R. Chemometric interpretation of digital image colorimetry. Application for titanium determination in plastics. Microchem. J. 2010, 96, 380-385.

25. MicroArray Profile for ImageJ. Available online: http://www.optinav.com/MicroArray_Profile.htm (accessed on 28 July 2012). 
26. Singleton, J.; Zentner, C.; Buser, J.; Yager, P.; LaBarre, P.; Weigl, B.H. Instrument-free exothermic heating with phase change temperature control for paper microfluidic devices. Proc. SPIE 2013, 8615,8615 .

27. Carrilho, E.; Phillips, S.T.; Vella, S.J.; Martinez, A.W.; Whitesides, G.M. Paper microzone plates. Anal. Chem. 2009, 81, 5990-5998.

28. Ramachandran, S.; Fu, E.; Lutz, B.; Yager, P. Long-term dry storage of an enzyme-based reagent system for ELISA in point-of-care devices. Analyst 2014, 139, 1456-1462.

(C) 2015 by the authors; licensee MDPI, Basel, Switzerland. This article is an open access article distributed under the terms and conditions of the Creative Commons Attribution license (http://creativecommons.org/licenses/by/4.0/). 